\title{
GLOBAL DYNAMICS ABOVE THE GROUND STATE ENERGY FOR THE ONE-DIMENSIONAL NLKG EQUATION
}

\author{
J. KRIEGER, K. NAKANISHI, AND W. SCHLAG
}

\begin{abstract}
In this paper we obtain a global characterization of the dynamics of even solutions to the one-dimensional nonlinear Klein-Gordon (NLKG) equation on the line with focusing nonlinearity $|u|^{p-1} u, p>5$, provided their energy exceeds that of the ground state only sightly. The method is the same as in the three-dimensional case [15], the major difference being in the construction of the center-stable manifold. The difficulty there lies with the weak dispersive decay of 1-dimensional NLKG. In order to address this specific issue, we establish local dispersive estimates for the perturbed linear Klein-Gordon equation, similar to those of Mizumachi [14]. The essential ingredient for the latter class of estimates is the absence of a threshold resonance of the linearized operator.
\end{abstract}

\section{INTRODUCTION}

In this paper we address the long-time evolution of solutions to the nonlinear Klein-Gordon equation

$$
u_{t t}-u_{x x}+u=|u|^{p-1} u
$$

in $1+1$ dimensions, and with $p>5$. It is standard that this equation is locally wellposed in the energy class $H^{1} \times L^{2}$ and can exhibit both global existence (for example for small data) as well as finite time blowup (for example, for negative energy). In addition, equation (1.1) admits explicit soliton solutions

$$
Q(x)=\alpha \cosh ^{-\frac{1}{\beta}}(\beta x), \quad \alpha=\left(\frac{p+1}{2}\right)^{\frac{1}{p-1}}, \quad \beta=\frac{p-1}{2}
$$

Our goal in this paper is to prove the following result, where

$$
E(u, \dot{u})=\int_{-\infty}^{\infty}\left[\frac{1}{2}\left(\left|\partial_{x} u\right|^{2}+|\dot{u}|^{2}+|u|^{2}\right)-\frac{1}{p+1}|u|^{p+1}\right] d x
$$

is the conserved energy.

Theorem 1.1. Let $p>5$. There exists $\varepsilon>0$ such that any even data $\left(u_{0}, u_{1}\right) \in$ $H^{1} \times L^{2}(\mathbb{R})$ with energy

$$
E(u, \dot{u})<E(Q, 0)+\varepsilon^{2}
$$

have the property that the solutions $u(t)$ of (1.1) associated with these data exhibit the following trichotomy:

- $u$ blows up in finite positive time

2010 Mathematics Subject Classification. 35L70, 35Q55.

Key words and phrases. nonlinear wave equation, ground state, hyperbolic dynamics, stable manifold, unstable manifold, scattering theory, blow up.

The third author was supported by the NSF, DMS-0617854, and a Guggenheim fellowship. 
- $u$ exists globally and scatters to zero as $t \rightarrow \infty$

- $u$ exists globally and scatters to $Q$, i.e., there exist $\left(v_{0}, v_{1}\right) \in H^{1} \times L^{2}$ with the property that

$$
(u(t), \dot{u}(t))=(Q, 0)+(v(t), \dot{v}(t))+o_{\mathcal{H}}(1) \quad t \rightarrow \infty
$$

for some free Klein-Gordon solution $(v(t), \dot{v}(t)) \in H^{1} \times L^{2}$.

In addition, the set of even data as in (1.4) splits into nine nonempty disjoint sets corresponding to all possible combinations of this trichotomy as $t \rightarrow \pm \infty$.

Moreover, we obtain a characterization of the threshold solutions, i.e., those with energies $E(\vec{u})=E(Q, 0)$, cf. [5], [6]. In fact, we find the following.

Corollary 1.2. The even solutions to (1.1) with energy $E(\vec{u})=E(Q, 0)$ can be characterized as follows:

- they blow up in both the positive and negative time directions

- the exist globally on $\mathbb{R}$ and scatter as $t \rightarrow \pm \infty$

- they are constant $\pm Q$

- they equal one of the following solutions, for some $t_{0} \in \mathbb{R}$ :

$\sigma W_{+}\left(t+t_{0}, x\right), \quad \sigma W_{-}\left(t+t_{0}, x\right), \quad \sigma W_{+}\left(-t+t_{0}, x\right), \quad \sigma W_{-}\left(-t+t_{0}, x\right)$ where $\left(W_{ \pm}(t, \cdot), \partial_{t} W_{ \pm}(t, \cdot)\right)$ approach $(Q, 0)$ exponentially fast in $\mathcal{H}$ as $t \rightarrow$ $\infty$, and $\sigma= \pm 1$. As $t \rightarrow-\infty, W_{+}$scatters to zero, whereas $W_{-}$blows up in finite time.

As usual, the image of $W_{ \pm}$and $Q$ form a one-dimensional stable manifold associated with $(Q, 0)$, cf. [2]. The unstable manifold is obtained by time-reversal. Moreover, the solutions in Theorem 1.1 which scatter to $Q$ form a $C^{1}$ manifold $^{1}$ in $\mathcal{H}$ of co-dimension 1 which is the center-stable manifold associated with $(Q, 0)$. The center manifold is obtained by the transverse intersection of the two center-stable manifolds corresponding to $t \rightarrow \pm \infty$, respectively. We remark that the case of energies $E(\vec{u})<E(Q, 0)$ was settled in [18]. There it was found that one either has global existence or blowup in both time directions. That global existence entails scattering to zero was only recently shown in [8].

The restriction $p>5$ in Theorem 1.1 appears to be a technical one; in fact, it seems reasonable to believe that Theorem 1.1 remains valid in the range $p>3$, and possibly even for some $p \leq 3$. At $p=3$ the linearized operator $L_{+}$has a threshold resonance which might affect the result. But more importantly, in the range $p<5$ small data scattering cannot be done on the basis of Strichartz estimates alone which is of course a serious obstacle at this point. The case $p=5$ is perhaps accessible, but we exclude it here (as in [8]). We remark that unlike the NLS case, the hyperbolic structure underlying our proof of Theorem 1.1 is still present for all $1<p \leq 5$. This refers to the fact that the structure of the spectrum of the linearized form of (1.1) around $Q$ does not change significantly.

This paper is closely related to [15], and for many details in the following section we refer the reader to that paper. The main difference lies with the construction of the center-stable manifold. The radial assumption in [15] was removed in [17], and we expect similarly that one can obtain Theorem 1.1 for all data, and not just

\footnotetext{
${ }^{1}$ It is in fact smoother than $C^{1}$, but we do not pursue this here.
} 
even ones; but we do not pursue that here. Finally, see [16] for similar results on three-dimensional cubic NLS, and [12] for the critical wave equations in three and five dimensions.

\section{Global existence Vs. Finite time Blowup}

In this section we recall the statement and proof of the "nine set theorem" from [15]. That theorem is weaker than Theorem 1.1 in the sense that "scattering to $Q$ " is replaced by "trapped by $Q$ ", which means that the solution remains in a small neighborhood of $Q$ for all times (positive or negative). In the following section we shall than take up the issue of proving that trapped by $Q$ actually implies the stronger scattering property. This amounts to proving the existence of a centerstable manifold as in [15]. It is at that point that this paper differs most from [15], due to the fact that the one-dimensional equation exhibits much less dispersion than the three-dimensional one. Let $\vec{u}=(u, \dot{u})$ and set

$$
\mathcal{H}^{\varepsilon}:=\left\{\vec{u} \in \mathcal{H}=H^{1} \times L^{2} \mid E(\vec{u})<E(Q, 0)+\varepsilon^{2}, \vec{u}(t, x)=\vec{u}(t,-x)\right\}
$$

Theorem 2.1. Consider all solutions of NLKG (1.1) with initial data $\vec{u}(0) \in \mathcal{H}^{\varepsilon}$ for some small $\varepsilon>0$. The solution set is decomposed into nine non-empty sets characterized as

(1) Scattering to 0 for both $t \rightarrow \pm \infty$,

(2) Finite time blow-up on both sides $\pm t>0$,

(3) Scattering to 0 as $t \rightarrow \infty$ and finite time blow-up in $t<0$,

(4) Finite time blow-up in $t>0$ and scattering to 0 as $t \rightarrow-\infty$,

(5) Trapped by $\pm Q$ for $t \rightarrow \infty$ and scattering to 0 as $t \rightarrow-\infty$,

(6) Scattering to 0 as $t \rightarrow \infty$ and trapped by $\pm Q$ as $t \rightarrow-\infty$,

(7) Trapped by $\pm Q$ for $t \rightarrow \infty$ and finite time blow-up in $t<0$,

(8) Finite time blow-up in $t>0$ and trapped by $\pm Q$ as $t \rightarrow-\infty$,

(9) Trapped by $\pm Q$ as $t \rightarrow \pm \infty$,

where "trapped by $\pm Q$ " means that the normalized solution stays in a $O(\varepsilon)$ neighborhood of $\pm Q$ forever after some time (or before some time). The initial data sets for (1)-(4), respectively, are open.

2.1. Hyperbolic and variational structures. We now recall the main steps in the three-dimensional proof from [15], which in essence carries over to the onedimensional case with only minimal changes. Next to the conserved energy $E$, we require the "static energy" or "action"

$$
J(u)=\int\left[\frac{1}{2}\left(\left|\partial_{x} u\right|^{2}+|u|^{2}\right)-\frac{1}{p+1}|u|^{p+1}\right] d x
$$

and the functionals

$$
\begin{aligned}
& K_{0}(u)=\left\langle J^{\prime}(u) \mid u\right\rangle=\int\left(\left|\partial_{x} u\right|^{2}+|u|^{2}-|u|^{p+1}\right) d x \\
& K_{2}(u)=\left\langle J^{\prime}(u) \mid A\right\rangle=\int\left(\left|\partial_{x} u\right|^{2}-\frac{p-1}{2(p+1)}|u|^{p+1}\right) d x
\end{aligned}
$$


with $A=\frac{1}{2}\left(x \partial_{x}+\partial_{x} x\right)$ the generator of dilations. It is standard that $\pm Q\left(\cdot+x_{0}\right)$ as in (1.2) are the only solutions of the static equation

$$
-\phi^{\prime \prime}+\phi=|\phi|^{p-1} \phi
$$

which are in $H^{1}$, and they uniquely minimize $J(\phi)$ subject to $K_{s}(\phi)=0, s=0,2$. Setting

$$
u=\sigma[Q+v], \quad v=\lambda \rho+\gamma, \quad \gamma \perp \rho
$$

where $\sigma= \pm$, one obtains

$$
E(\vec{u})=J(Q)+\frac{1}{2}\left(\dot{\lambda}^{2}-k^{2} \lambda^{2}\right)+\frac{1}{2}\left(\left\langle L_{+} \gamma \mid \gamma\right\rangle+\|\dot{\gamma}\|_{2}^{2}\right)-C(v)
$$

where $C(v)=O\left(\|v\|_{H^{1}}^{3}\right)$. We now define

$$
\begin{aligned}
\|v\|_{E}^{2} & :=\frac{1}{2}\left(\dot{\lambda}^{2}+k^{2} \lambda^{2}\right)+\frac{1}{2}\left(\left\langle L_{+} \gamma \mid \gamma\right\rangle+\|\dot{\gamma}\|_{2}^{2}\right) \\
d_{\sigma}(\vec{u}) & :=\sqrt{\|\vec{v}\|_{E}^{2}-\chi\left(\|\vec{v}\|_{E} /\left(2 \delta_{E}\right)\right) C(V)}
\end{aligned}
$$

where $0<\delta_{E} \ll 1$ and $\chi$ is a suitable cut-off function so that

$$
\begin{gathered}
\|\vec{v}\|_{E} / 2 \leq d_{\sigma}(\vec{u}) \leq 2\|\vec{v}\|_{E}, \quad d_{\sigma}(\vec{u})=\|\vec{v}\|_{E}+O\left(\|\vec{v}\|_{E}^{2}\right), \\
d_{\sigma}(\vec{u}) \leq \delta_{E} \Longrightarrow d_{\sigma}(\vec{u})^{2}=E(\vec{u})-J(Q)+k^{2} \lambda^{2} .
\end{gathered}
$$

The eigenmode dominance and the ejection process from [15] carry over to this setting without changes. For the sake of completeness, we reproduce these statements here, but refer the reader to [15] for the proofs.

Lemma 2.2. For any $\vec{u} \in \mathcal{H}$ satisfying

$$
E(\vec{u})<J(Q)+d_{Q}(\vec{u})^{2} / 2, \quad d_{Q}(\vec{u}) \leq \delta_{E}
$$

one has $d_{Q}(\vec{u}) \simeq|\lambda|$.

The following ejection lemma is most important in the proof of Theorem 2.1 and Theorem 1.1. It states that a solution which penetrates a small neighborhood of $\pm(Q, 0)$ deep enough (compared to $\varepsilon$ ) in $\mathcal{H}^{\varepsilon}$, but which is not trapped by these equilibria, is necessary ejected from the neighborhood via the exponentially increasing mode. The nontrapping here is formulated via the condition $d_{Q}(\vec{u}(t)) \geq d_{Q}(\vec{u}(0))$ for small $t \geq 0$, cf. (2.10).

Lemma 2.3. There exists a constant $0<\delta_{X} \leq \delta_{E}$ with the following property. Let $u(t)$ be a local solution of $N L K G$ on an interval $[0, T]$ satisfying

$$
R:=d_{Q}(\vec{u}(0)) \leq \delta_{X}, \quad E(\vec{u})<J(Q)+R^{2} / 2
$$

and for some $t_{0} \in(0, T)$,

$$
d_{Q}(\vec{u}(t)) \geq R \quad\left(0<\forall t<t_{0}\right)
$$


Then $d_{Q}(\vec{u}(t))$ increases monotonically until reaching $\delta_{X}$, and meanwhile,

$$
\begin{aligned}
& d_{Q}(\vec{u}(t)) \simeq-\mathfrak{s} \lambda(t) \simeq-\mathfrak{s} \lambda_{+}(t) \simeq e^{k t} R, \\
& \left|\lambda_{-}(t)\right|+\|\vec{\gamma}(t)\|_{E} \lesssim R+e^{2 k t} R^{2}, \\
& \min _{s=0,2} \mathfrak{s} K_{s}(u(t)) \gtrsim d_{Q}(\vec{u}(t))-C_{*} d_{Q}(\vec{u}(0)),
\end{aligned}
$$

for either $\mathfrak{s}=+1$ or $\mathfrak{s}=-1$, where $C_{*} \geq 1$ is a constant.

One point in which the proofs depend on the dimension and the power of the nonlinearity is the linearization of the $K$ functionals. Here they are

$$
\begin{aligned}
& K_{0}(Q+v)=-(p-1)\left\langle Q^{p} \mid v\right\rangle+O\left(\|v\|_{H^{1}}^{2}\right) \\
& K_{2}(Q+v)=-\left\langle\frac{p-5}{2} Q^{p}+2 Q \mid v\right\rangle+O\left(\|v\|_{H^{1}}^{2}\right)
\end{aligned}
$$

Next, we require the following variational lower bounds, which are proved as in dimension three (but one needs to use the concentration compactness principle as in [17]).

Lemma 2.4. For any $\delta>0$, there exist $\varepsilon_{0}(\delta), \kappa_{0}, \kappa_{1}(\delta)>0$ such that for any $\vec{u} \in \mathcal{H}$ satisfying

$$
E(\vec{u})<J(Q)+\varepsilon_{0}(\delta)^{2}, \quad d_{Q}(\vec{u}) \geq \delta
$$

one has either

$$
K_{0}(u) \leq-\kappa_{1}(\delta) \quad \text { and } \quad K_{2}(u) \leq-\kappa_{1}(\delta)
$$

or

$$
K_{0}(u) \geq \min \left(\kappa_{1}(\delta), \kappa_{0}\|u\|_{H^{1}}^{2}\right) \quad \text { and } \quad K_{2}(u) \geq \min \left(\kappa_{1}(\delta), \kappa_{0}\left\|\partial_{x} u\right\|_{L^{2}}^{2}\right) .
$$

Combining the signs of $\lambda$ and $K_{s}$ one now obtains a global sign functional.

Lemma 2.5. Let $\delta_{S}:=\delta_{X} /\left(2 C_{*}\right)>0$ where $\delta_{X}$ and $C_{*} \geq 1$ are constants from Lemma 2.3. Let $0<\delta \leq \delta_{S}$ and

$$
\mathcal{H}_{(\delta)}:=\left\{\vec{u} \in \mathcal{H} \mid E(\vec{u})<J(Q)+\min \left(d_{Q}(\vec{u})^{2} / 2, \varepsilon_{0}(\delta)^{2}\right)\right\},
$$

where $\varepsilon_{0}(\delta)$ is given by Lemma 2.4. Then there exists a unique continuous function $\mathfrak{S}: \mathcal{H}_{(\delta)} \rightarrow\{ \pm 1\}$ satisfying

$$
\left\{\begin{array}{l}
\vec{u} \in \mathcal{H}_{(\delta)}, d_{Q}(\vec{u}) \leq \delta_{E} \quad \Longrightarrow \mathfrak{S}(\vec{u})=-\operatorname{sign} \lambda, \\
\vec{u} \in \mathcal{H}_{(\delta)}, d_{Q}(\vec{u}) \geq \delta \quad \Longrightarrow \mathfrak{S}(\vec{u})=\operatorname{sign} K_{0}(u)=\operatorname{sign} K_{2}(u),
\end{array}\right.
$$

where we set $\operatorname{sign} 0=+1$ (a convention for the case $u=0)$.

The free energy is uniformly bounded in the region $\mathfrak{S}=1$. More precisely, one has the following result.

Lemma 2.6. There exists $M_{*} \sim J(Q)^{1 / 2}$ such that for any $\vec{u} \in \mathcal{H}_{\left(\delta_{S}\right)}$ satisfying $\mathfrak{S}(\vec{u})=+1$ we have $\|\vec{u}\|_{\mathcal{H}} \leq M_{*}$.

For the proofs of these statements, see [15]. 
2.2. Absence of almost homoclinic orbits. In the section, we recall the onepass theorem from [15]. We present the proof of this result for the sake of completeness. We begin with the following result on global scattering which excludes the degenerate case of $K_{2}(u(t))>0$ being to small for too long.

Lemma 2.7. For any $M>0$, there exists $\mu_{0}(M)>0$ with the following property. Let $u(t)$ be a finite energy solution of $N L K G(1.1)$ on $[0,2]$ satisfying

$$
\|\vec{u}\|_{L_{t}^{\infty}(0,2 ; \mathcal{H})} \leq M, \quad \int_{0}^{2}\left\|\partial_{x} u(t)\right\|_{L^{2}}^{2} d t \leq \mu^{2}
$$

for some $\mu \in\left(0, \mu_{0}\right]$. Then $u$ extends to a global solution and scatters to 0 as $t \rightarrow \pm \infty$, and moreover $\|u(t)\|_{L_{t}^{p} L_{x}^{2 p}\left(\mathbb{R} \times \mathbb{R}^{3}\right)} \ll \mu^{\kappa}$ for some constant $\kappa=\kappa(p)>0$.

Proof. First we see that $u$ can be approximated by the free solution

$$
v(t):=e^{i\langle\nabla\rangle t} v_{+}+e^{-i\langle\nabla\rangle t} v_{-}, \quad v_{ \pm}:=\left[u(0) \mp i\langle\nabla\rangle^{-1} \dot{u}(0)\right] / 2 .
$$

This follows simply from the Duhamel formula

$$
\begin{aligned}
\|v-u\|_{L_{t}^{\infty} H_{x}^{1}(0,2)} & \lesssim\left\||u|^{p-1} u\right\|_{L_{t}^{1}\left((0,2), L_{x}^{2}\right)} \lesssim\|u\|_{L_{t}^{p} L_{x}^{2 p}}^{p} \\
& \lesssim\left\|\partial_{x} u\right\|_{L_{t}^{2}\left((0,2), L_{x}^{2}\right)}^{2}\|u\|_{L_{t}^{\infty}((0,2), \mathcal{H})}^{p-2} \leq \mu^{2} M^{p-2} \ll \mu,
\end{aligned}
$$

if $\mu_{0} M^{p-2} \ll 1$, where we used Hölder's inequality, Sobolev embedding, and $p \geq 5$. In particular,

$$
\begin{aligned}
4 \mu^{2} & \geq \int_{0}^{2}\left\|\partial_{x} v(t)\right\|_{L_{x}^{2}}^{2} d t \\
& =C \int|\xi|^{2}\left[2\left|\widehat{v}_{+}\right|^{2}+2\left|\widehat{v}_{-}\right|^{2}+\operatorname{Im}\left\{\langle\xi\rangle^{-1}\left(e^{4 i\langle\xi\rangle}-1\right) \widehat{v}_{+} \widehat{\widehat{v}}_{-}\right\}\right] d \xi \\
& \gtrsim\left\|\partial_{x} v_{+}\right\|_{L^{2}}^{2}+\left\|\partial_{x} v_{-}\right\|_{L^{2}}^{2},
\end{aligned}
$$

where $\widehat{v}$ denotes the Fourier transform in $x$ of $v$. Now we use the Strichartz estimate for the free Klein-Gordon equation

$$
\left\|e^{ \pm i\langle\nabla\rangle t} \varphi\right\|_{L_{t}^{p} B_{q, 2}^{\alpha}} \lesssim\|\varphi\|_{H_{x}^{1}}
$$

with $\alpha=1-\frac{3}{p}, \frac{1}{q}=\frac{1}{2}-\frac{2}{p}$, see (3.39) below. Combining it with Sobolev, we obtain with $p>5$ fixed, $\alpha, q$ as in (2.18), and $\beta=\frac{1}{2}\left(1-\frac{5}{p}\right)>0$,

$$
\|v\|_{L_{t}^{p} L_{x}^{2 p}(\mathbb{R} \times \mathbb{R})} \lesssim\|v\|_{L_{t}^{p} \dot{B}_{q, 2}^{\beta}(\mathbb{R} \times \mathbb{R})} \lesssim \sum_{ \pm}\left\|v_{ \pm}\right\|_{\dot{H}^{\beta} \cap \dot{H}^{1-(\alpha-\beta)}} \ll \mu^{\kappa}
$$

if $\mu_{0}$ is sufficiently small depending on $M$. Therefore, we can identify $u$ as the fixed point for the iteration in the global Strichartz norm

$$
\|u\|_{L_{t}^{\infty} H_{x}^{1}(\mathbb{R} \times \mathbb{R})} \lesssim M, \quad\|u\|_{L_{t}^{p} L_{x}^{2 p}(\mathbb{R} \times \mathbb{R})} \ll \mu^{\kappa},
$$

which automatically scatters.

Using the constants in Lemmas 2.3-2.7, we choose $\varepsilon_{*}, \delta_{*}, R_{*}, \mu>0$ such that

$$
\begin{aligned}
& \delta_{*} \leq \delta_{S}, \quad \delta_{*} \ll \delta_{X}, \quad \varepsilon_{*} \leq \varepsilon_{0}\left(\delta_{*}\right), \\
& \varepsilon_{*} \ll R_{*} \ll \min \left(\delta_{*}, \kappa_{1}\left(\delta_{*}\right)^{1 / 2}, \kappa_{0}^{1 / 2} \mu, J(Q)^{1 / 2}\right),
\end{aligned}
$$




$$
\mu<\mu_{0}\left(M_{*}\right), \quad \mu^{\kappa} \ll J(Q)^{1 / 2} .
$$

Suppose that a solution $u(t)$ on the maximal existence interval $I \subset \mathbb{R}$ satisfies for some $\varepsilon \in\left(0, \varepsilon_{*}\right], R \in\left(2 \varepsilon, R_{*}\right]$, and $\tau_{1}<\tau_{2}<\tau_{3} \in I$,

$$
E(\vec{u})<J(Q)+\varepsilon^{2}, \quad d_{Q}\left(\vec{u}\left(\tau_{1}\right)\right)<R<d_{Q}\left(\vec{u}\left(\tau_{2}\right)\right)>R>d_{Q}\left(\vec{u}\left(\tau_{3}\right)\right) .
$$

Then there exist $T_{1} \in\left(\tau_{1}, \tau_{2}\right)$ and $T_{2} \in\left(\tau_{2}, \tau_{3}\right)$ such that

$$
d_{Q}\left(\vec{u}\left(T_{1}\right)\right)=R=d_{Q}\left(\vec{u}\left(T_{2}\right)\right) \leq d_{Q}(\vec{u}(t)) \quad\left(T_{1}<t<T_{2}\right) .
$$

Lemma 2.5 gives us a fixed sign

$$
\{ \pm 1\} \ni \mathfrak{s}:=\mathfrak{S}(u(t)) \quad\left(T_{1}<t<T_{2}\right)
$$

Based on the exact same virial identity as in [15], viz.

$$
V_{w}(t):=\left\langle w u_{t} \mid(x \nabla+\nabla x) u\right\rangle, \quad \dot{V}_{w}(t)=-K_{2}(u(t))+\text { error }
$$

with some suitable time-dependent cut-off $w$ (for which the error is controlled by the energy outside of some space-time rhombus) we now obtain the following result.

Theorem 2.8 (One-pass theorem). Let $\varepsilon_{*}, R_{*}>0$ be as in (2.19). If a solution $u$ of $N L K G$ on an interval I satisfies for some $\varepsilon \in\left(0, \varepsilon_{*}\right], R \in\left(2 \varepsilon, R_{*}\right]$, and $\tau_{1}<\tau_{2} \in I$,

$$
E(\vec{u})<J(Q)+\varepsilon^{2}, \quad d_{Q}\left(\vec{u}\left(\tau_{1}\right)\right)<R=d_{Q}\left(\vec{u}\left(\tau_{2}\right)\right)
$$

then for all $t \in\left(\tau_{2}, \infty\right) \cap I=: I^{\prime}$, we have $d_{Q}(\vec{u}(t))>R$.

Moreover, there exist disjoint subintervals $I_{1}, I_{2}, \cdots \subset I^{\prime}$ with the following property: On each $I_{m}$, there exists $t_{m} \in I_{m}$ such that

$$
d_{Q}(\vec{u}(t)) \simeq e^{k\left|t-t_{m}\right|} d_{Q}\left(\vec{u}\left(t_{m}\right)\right), \quad \min _{s=0,2} \mathfrak{s} K_{s}(u(t)) \gtrsim d_{Q}(\vec{u}(t))-C_{*} d_{Q}\left(\vec{u}\left(t_{m}\right)\right),
$$

where $\mathfrak{s}:=\mathfrak{S}(\vec{u}(t)) \in\{ \pm 1\}$ is constant, $d_{Q}(\vec{u}(t))$ is increasing for $t>t_{m}$, decreasing for $t<t_{m}$, equals to $\delta_{X}$ on $\partial I_{m}$. For each $t \in I^{\prime} \backslash \bigcup_{m} I_{m}$ and $s=0,2$, one has $(t-1, t+1) \subset I^{\prime}, d_{Q}(\vec{u}(t)) \geq \delta_{*}$ and

$$
\int_{t-1}^{t+1} \min _{s=0,2} \mathfrak{s} K_{s}\left(u\left(t^{\prime}\right)\right) d t^{\prime} \gg R_{*}^{2} .
$$

By the monotonicity, we can keep applying the above theorem at each $t>\tau_{2}$ until $d_{Q}(\vec{u})$ reaches $R_{*}$. Besides, one concludes that at any later time $t_{m}>\tau_{2}$ necessarily $d_{Q}(\vec{u})>R_{*}$. In other words, $u$ cannot return to the distance $R_{*}$ to $\pm Q$, after it is ejected to the distance $\delta_{X}>R_{*}$.

2.3. Blowup in $\mathfrak{S}=-1$. If follows from the one-pass theorem that the sign $\mathfrak{S}$ stabilizes, i.e., if $u(t)$ exists on some maximal time-interval $I=\left[0, T_{*}\right)$, then there is $0<T_{0} \in I$ with the property that $\mathfrak{S}(\vec{u}(t))=1$ or -1 on $T_{0}<t<T_{*}$. We now show by the usual Payne-Sattinger convexity argument that necessarily $T_{*}<\infty$ if $\mathfrak{S}(\vec{u}(t))=-1$.

Suppose not, and define $y(t)=\|u(t)\|_{2}^{2}$. Then

$$
\ddot{y}(t)=2\left[\|\dot{u}\|_{2}^{2}-K_{0}(u(t))\right]
$$


implies together with the uniform negative upper bound on $K_{0}(u(t))$ for large times that $y(t) \rightarrow \infty$ as $t \rightarrow \infty$. In particular,

$$
\ddot{y}(t)=-2(p+1) E(\vec{u})+2 p\|\dot{u}(t)\|_{2}^{2}+p\|u(t)\|_{H^{1}}^{2} \geq 2 p\|\dot{u}(t)\|_{2}^{2}
$$

for large times, whence also, with $\alpha=\frac{p}{2}-1$,

$$
\frac{d^{2}}{d t^{2}} y^{-\alpha}=-\alpha y^{-\alpha-2}\left(y \ddot{y}-(\alpha+1) \dot{y}^{2}\right)<0
$$

for large times. But this is a contradiction to $y(t) \rightarrow \infty$.

2.4. Global existence and scattering in $\mathfrak{S}=1$. It is clear that $T_{*}=+\infty$ if $\mathfrak{S}=1$. Indeed, this follows from the fact that $K_{0}(u(t)) \geq 0$ forces

$$
E(\vec{u}) \simeq\|u(t)\|_{H^{1}}^{2}+\|\dot{u}(t)\|_{2}^{2}
$$

for all times. On the other hand, scattering is harder but can be obtained via the Kenig-Merle by essentially the exact same arguments as in [15]. The only difference is that the Bahouri-Gerard [3] (as well as Merle-Vega [13]) decomposition and the perturbation lemma need to be stated in terms of Strichartz norms of the 1-dimensional equation. But this has already been done in [8], and we see no reason to write this out again. Hence, we now obtain the following scattering result in this case.

Theorem 2.9. For each $\varepsilon \in\left(0, \varepsilon_{*}\right]$, there exists $0<M\left(J(Q)+\varepsilon^{2}\right)<\infty$ such that if a solution $u$ of $N L K G$ on $[0, \infty)$ satisfies $E(\vec{u}) \leq J(Q)+\varepsilon^{2}, d_{Q}(\vec{u}(t)) \geq R_{*}$ and $\mathfrak{S}(\vec{u}(t))=+1$ for all $t \geq 0$, then $u$ scatters to 0 as $t \rightarrow \infty$ and $\|u\|_{L_{t}^{p} L_{x}^{2 p}(0, \infty)} \leq M$.

The proof of Theorem 2.1 now follows by the exact same arguments as in [15].

\section{Perturbation theory near the soliton For EVEn SOlutions}

The goal of this section is to construct the center-stable manifold near $Q$ for even solutions of the NLKG equation (1.1). Due to the lack of adequate dispersion in one dimension, we will need to invoke weighted estimates in our construction similar to those appearing in [14]. To formulate the theorem, we recall the basic hyperbolic/dispersive dynamics near the soliton. Linearizing (1.1) around $Q$ yields the operator

$$
L_{+}=-\partial_{x x}+1-p Q^{p-1}
$$

which has a ground state $L_{+} \rho=-k^{2} \rho, k>0, \rho>0$, a zero eigenvalue $L_{+} \partial_{x} Q=0$, no other eigenvalues, and no threshold resonance (provided $p>3$, which is the sharp condition for these properties), see for example [11, Lemma 9.1]. Similarly,

$$
L_{-}=-\partial_{x x}+1-Q^{p-1}
$$

has no negative spectrum, $Q$ as a ground state since $L_{-} Q=0$, and no other eigenvalues, and no threshold resonance (again for $p>3$ ).

We now seek solutions of the form ${ }^{2} u=Q+v=Q+\mu \rho+w$ with $v$ small, and $w \perp \rho$. We write $\vec{u}=(u, \dot{u})$.

\footnotetext{
${ }^{2}$ In contrast to the previous section we use $\mu$ here since $\lambda$ will be used for the spectral parameter.
} 
Proposition 3.1 (Center-Stable manifolds). Let $p \geq 5$. There exists $\nu>0$ small and a $C^{1}$ graph $\mathcal{M}$ in $B_{\nu}(Q, 0) \subset \mathcal{H}$ so that $(Q, 0) \in \mathcal{M}$, with tangent plane

$$
T_{Q} \mathcal{M}=\left\{\left(u_{0}, u_{1}\right) \in \mathcal{H} \mid\left\langle k u_{0}+u_{1} \mid \rho\right\rangle=0\right\}
$$

at $(Q, 0)$ in the sense that

$$
\sup _{x \in \partial B_{\delta}(Q, 0)} \operatorname{dist}\left(x, T_{Q} \mathcal{M}\right) \lesssim \delta^{2} \quad \forall 0<\delta<\nu
$$

Any data $\left(u_{0}, u_{1}\right) \in \mathcal{M}$ lead to global evolutions of (1.1) of the form $u=Q+v=$ $Q+\mu \rho+w$ where $v$ satisfies

$$
\|(v, \dot{v})\|_{L_{t}^{\infty} \mathcal{H}}+\|v\|_{L^{p}\left((0, \infty) ; L^{2 p}(\mathbb{R})\right)} \lesssim \nu
$$

and scatters to a free Klein-Gordon solution in $\mathcal{H}$, i.e., there exists a unique free Klein-Gordon solution $w_{\infty}$ such that

$$
|\mu(t)|+|\dot{\mu}(t)|+\left\|\vec{w}(t)-\vec{w}_{\infty}(t)\right\|_{\mathcal{H}} \rightarrow 0,
$$

as $t \rightarrow \infty$. In particular, we have $E(\vec{u})=J(Q)+\left\|\vec{w}_{\infty}\right\|_{\mathcal{H}}^{2} / 2$. Finally, any solution that remains inside $B_{\nu}(Q, 0)$ for all $t \geq 0$ necessarily lies entirely on $\mathcal{M}$, and $\mathcal{M}$ is invariant under the flow for all $t \geq 0$.

The regularity of the graph $\mathcal{M}$ is better than $C^{1}$ (depending on $p$ ), but for general $p$ the nonlinearity is not smooth and one can therefore not expect $\mathcal{M}$ to be smooth (if the nonlinearity is given by an integer power such as $p=5,7$ then $\mathcal{M}$ is smooth). The remainder of this section is devoted to proving this result. As in [14], [4], we shall use weighted $L_{x}^{\infty} L_{t}^{2}$ bounds to overcome the weaker dispersion in one dimension. These estimates hinge on the fact that $L_{ \pm}$have no threshold resonance which is reflected by the regularity of the spectral measure near zero energy. The latter then guarantees faster local decay of the Klein-Gordon evolutions relative to $L_{ \pm}$. To obtain these bounds we shall use the distorted Fourier transform.

3.1. The distorted Fourier transform. We begin by recalling the distorted Fourier transform relative to a Schrödinger operator on the line

$$
\mathcal{L}:=-\frac{d^{2}}{d x^{2}}+V
$$

with real-valued potential. In our case

$$
V(x)=-\alpha \cosh ^{-2}(\beta x)
$$

for suitable $\alpha, \beta>0$, but for the moment we only need $V \in L_{\text {loc }}^{1}(\mathbb{R})$ and $\mathcal{L}$ in the limit-point case at $\pm \infty$. This material is of course standard, see for example Section 2 of [7]. Define $\phi_{\alpha}\left(x, x_{0} ; z\right), \theta_{\alpha}\left(x, x_{0} ; z\right)$ to be the fundamental system of solutions of

$$
\mathcal{L} \psi=z \psi, \quad z \in \mathbb{C}
$$

so that

$$
\begin{aligned}
& \phi_{\alpha}\left(x_{0}, x_{0} ; z\right)=-\theta_{\alpha}^{\prime}\left(x_{0}, x_{0} ; z\right)=-\sin \alpha \\
& \phi_{\alpha}^{\prime}\left(x_{0}, x_{0} ; z\right)=\theta_{\alpha}\left(x_{0}, x_{0} ; z\right)=\cos \alpha
\end{aligned}
$$

where $x_{0} \in \mathbb{R}$ and $\alpha \in[0, \pi)$. Their Wronskian is

$$
W\left(\theta_{\alpha}, \phi_{\alpha}\right)=1
$$


The Weyl-Titchmarsh solutions are defined as the unique solutions $\psi_{ \pm, \alpha}\left(\cdot, x_{0} ; z\right) \in$ $L^{2}\left(\left[x_{0}, \pm \infty\right), d x\right)$ for $z \in \mathbb{C} \backslash \mathbb{R}$ which satisfy the boundary condition

$$
\psi_{ \pm}^{\prime}\left(x_{0}, x_{0} ; z\right) \sin \alpha+\psi_{ \pm}\left(x_{0}, x_{0} ; z\right) \cos \alpha=1
$$

This boundary condition ensures that

$$
\psi_{ \pm, \alpha}\left(x, x_{0} ; z\right)=\theta_{\alpha}\left(x, x_{0} ; z\right)+m_{ \pm, \alpha}\left(z, x_{0}\right) \phi_{\alpha}\left(x, x_{0} ; z\right)
$$

and the Wronskian

$$
W\left(\psi_{+}\left(\cdot, x_{0} ; z\right), \psi_{-}\left(\cdot, x_{0} ; z\right)\right)=m_{-, \alpha}\left(z, x_{0}\right)-m_{+, \alpha}\left(z, x_{0}\right)
$$

The Weyl-Titchmarsh functions $m_{ \pm, \alpha}$ are Herglotz functions, and the associated Weyl-Titchmarsh matrix

$$
M_{\alpha}\left(z, x_{0}\right):=\left[\begin{array}{cc}
\frac{1}{m_{-, \alpha}\left(z, x_{0}\right)-m_{+, \alpha}\left(z, x_{0}\right)} & \frac{1}{2} \frac{m_{-, \alpha}\left(z, x_{0}\right)+m_{+, \alpha}\left(z, x_{0}\right)}{m_{-, \alpha}\left(z, x_{0}\right)-m_{+, \alpha}\left(z, x_{0}\right)} \\
\frac{1}{2} \frac{m_{-, \alpha}\left(z, x_{0}\right)+m_{+, \alpha}\left(z, x_{0}\right)}{m_{-, \alpha}\left(z, x_{0}\right)-m_{+, \alpha}\left(z, x_{0}\right)} & \frac{m_{-, \alpha}\left(z, x_{0}\right) m_{+, \alpha}\left(z, x_{0}\right)}{m_{-, \alpha}\left(z, x_{0}\right)-m_{+, \alpha}\left(z, x_{0}\right)}
\end{array}\right]
$$

is a Herglotz matrix. This implies that there exists a nonnegative $2 \times 2$-matrix valued measure $\Omega_{\alpha}\left(d \lambda, x_{0}\right)$ so that the representation

$$
\begin{aligned}
M_{\alpha}\left(z, x_{0}\right) & =C_{\alpha}\left(x_{0}\right)+\int_{\mathbb{R}}\left[\frac{1}{\lambda-z}-\frac{\lambda}{1+\lambda^{2}}\right] \Omega_{\alpha}\left(d \lambda, x_{0}\right) \\
C_{\alpha}\left(x_{0}\right) & =C_{\alpha}\left(x_{0}\right)^{*}, \quad \int_{\mathbb{R}} \frac{\left\|\Omega_{\alpha}\left(d \lambda, x_{0}\right)\right\|}{1+\lambda^{2}}<\infty
\end{aligned}
$$

holds. The measure $\Omega_{\alpha}$ satisfies

$$
\Omega_{\alpha}\left(\left(\lambda_{1}, \lambda_{2}\right], x_{0}\right)=\pi^{-1} \lim _{\delta \rightarrow 0+} \lim _{\varepsilon \rightarrow 0+} \int_{\lambda_{1}+\delta}^{\lambda_{2}+\delta} \operatorname{Im} M_{\alpha}\left(\lambda+i \varepsilon, x_{0}\right) d \lambda
$$

The matrix measure $\Omega_{\alpha}$ plays the role of the spectral measure, as can be seen from the following Fourier representation relative to $\mathcal{L}$.

Proposition 3.2. Let $\alpha \in[0, \pi), f, g \in C_{0}^{\infty}(\mathbb{R}), F \in C(\mathbb{R}) \cap L^{\infty}(\mathbb{R})$, and $\lambda_{1}<\lambda_{2}$. Then

$$
\begin{aligned}
\left(f, F(H) E_{H}\left(\left(\lambda_{1}, \lambda_{2}\right]\right) g\right)_{L^{2}(\mathbb{R} ; d x)} & =\left(\widehat{f}_{\alpha}\left(\cdot, x_{0}\right), M_{F} M_{\chi_{\left(\lambda_{1}, \lambda_{2}\right]}} \widehat{g}_{\alpha}\left(\cdot, x_{0}\right)\right)_{L^{2}\left(\mathbb{R}, \Omega_{\alpha}\left(\cdot, x_{0}\right)\right)} \\
& =\int_{\left(\lambda_{1}, \lambda_{2}\right]}{\widehat{\hat{f}_{\alpha}}\left(\lambda, x_{0}\right)}^{T} \Omega_{\alpha}\left(d \lambda, x_{0}\right) \widehat{g}_{\alpha}\left(\lambda, x_{0}\right) F(\lambda)
\end{aligned}
$$

where

$$
\begin{aligned}
\widehat{h}_{\alpha, 1}\left(\lambda, x_{0}\right) & =\int_{\mathbb{R}} h(x) \theta_{\alpha}\left(x, x_{0} ; \lambda\right) d x, \quad \widehat{h}_{\alpha, 2}\left(\lambda, x_{0}\right)=\int_{\mathbb{R}} h(x) \phi_{\alpha}\left(x, x_{0} ; \lambda\right) d x \\
\widehat{h}_{\alpha}\left(\lambda, x_{0}\right) & =\left(\widehat{h}_{\alpha, 1}, \widehat{h}_{\alpha, 2}\right)^{T}\left(\lambda, x_{0}\right)
\end{aligned}
$$

This Fourier transform establishes a unitary correspondence between $L^{2}(\mathbb{R}, d x)$ and $L^{2}\left(\mathbb{R}, \Omega_{0}\right)$. 
For example, for the free case one finds that, with $\alpha=0, x_{0}=0$,

$$
\begin{aligned}
\phi_{0}(x, 0 ; \lambda) & =\frac{\sin \left(\lambda^{\frac{1}{2}} x\right)}{\lambda^{\frac{1}{2}}}, \quad \theta_{0}(x, 0 ; \lambda)=\cos \left(\lambda^{\frac{1}{2}} x\right) \\
m_{ \pm, 0}(z, 0) & = \pm i z^{\frac{1}{2}}, \quad z \in \mathbb{C} \backslash[0, \infty) \\
\Omega_{0}(d \lambda, 0) & =\frac{1}{2 \pi} \chi_{(0, \infty)}(\lambda)\left[\begin{array}{cc}
\lambda^{-\frac{1}{2}} & 0 \\
0 & \lambda^{\frac{1}{2}}
\end{array}\right] d \lambda
\end{aligned}
$$

This can be seen to lead to the usual Fourier transform on the line, but written as sin, cos transform, with the Fourier variable in the positive half-axis.

3.2. The wave operators. Throughout this section, we will assume for simplicity that the potential $V$ in (3.6) is a Schwartz function. Although much less is required for the following results to hold, it will be sufficient for our purposes to do so (since the soliton is a Schwartz function). Recall that the wave operators

$$
W_{ \pm}=s-\lim _{t \rightarrow \pm \infty} e^{i t \mathcal{L}} e^{i t \partial_{x}^{2}}
$$

exist and are isometries $L^{2}(\mathbb{R}) \rightarrow P_{c}(\mathcal{L}) L^{2}(\mathbb{R})$. Moreover, $W_{ \pm} W_{ \pm}^{*}=P_{c}(\mathcal{L}), W_{ \pm}^{*} W_{ \pm}=$ Id, and

$$
W_{ \pm}^{*} E_{\mathcal{L}} W_{ \pm}=E_{0}
$$

as an identity between projection valued measures, where $E_{0}, E_{\mathcal{L}}$ are the spectral resolutions of $-\partial_{x}^{2}$ and $\mathcal{L}$, respectively. One refers to (3.16) as the "intertwining property". It is equivalent to the statement that

$$
f(\mathcal{L}) P_{c}(\mathcal{L})=W_{ \pm} f\left(-\partial_{x}^{2}\right) W_{ \pm}^{*}
$$

for every continuous, bounded $f$ on the line. Weder [19] and Artbazar, Yajima [1], proved that under our assumptions, the wave operators $W_{ \pm}$are bounded on all $W^{k, p}, k \geq 0,1<p<\infty$. This is very useful, as it allows one to transfer estimates from the free case to $\mathcal{L}$ by means of (3.17). For example, one has the following multiplier result.

Lemma 3.3. Let $m:(0, \infty) \rightarrow \mathbb{C}$ satisfy

$$
\sup _{\lambda>0}\left[|m(\lambda)|+\lambda\left|m^{\prime}(\lambda)\right|\right] \leq C
$$

Then $m$ is a bounded Fourier multiplier on $P_{c}(\mathcal{L}) L^{p}(\mathbb{R})$ for $1<p<\infty$. By "Fourier multiplier" we mean a function $m(\lambda)$ that multiplies both components of the vector $\widehat{h}_{\alpha}$ in Proposition 3.2.

Proof. With $\mathcal{F}$ denoting the distorted, and $\mathcal{F}_{0}$ the free (as in (3.15)) Fourier transforms, respectively, one has

$$
\mathcal{F}^{-1} \circ \mathcal{M}_{m} \circ \mathcal{F} P_{c}(\mathcal{L})=W_{ \pm} \circ \mathcal{F}_{0}^{-1} \circ \mathcal{M}_{m} \circ \mathcal{F}_{0} \circ W_{ \pm}^{*}
$$

where $\mathcal{M}_{m}$ is the component-wise multiplication operator by $m(\lambda)$. 
3.3. Specializing to $L_{ \pm}$. Now we consider the case of $\mathcal{L}:=L_{ \pm}-1=-\frac{d^{2}}{d x^{2}}+V$ where $V$ is as in (3.7). In fact, we shall only use here that $V$ is an even Schwartz function and that $\mathcal{L}$ has no zero energy resonance. Recall that this means that there is no globally bounded solution of $\mathcal{L} f=0$. Note that the free case does have a zero energy resonance, as does

$$
\mathcal{L}=-\frac{d^{2}}{d x^{2}}-6 \cosh ^{-2}(x)
$$

which is the operator one obtains from linearizing the cubic NLKG equation.

Lemma 3.4. Suppose that $\mathcal{L}$ as in Proposition 3.2 has an even Schwartz potential and no zero energy resonance. Then the spectral measure $\Omega_{0}$ is diagonal, absolutely continuous on $(0, \infty)$, and of the form

$$
\begin{aligned}
& \Omega_{0}(d \lambda, 0)=\operatorname{diag}\left(O\left(\lambda^{\frac{1}{2}}\right), O\left(\lambda^{\frac{1}{2}}\right)\right) d \lambda, \quad \lambda \rightarrow 0 \\
& \Omega_{0}(d \lambda, 0)=\operatorname{diag}\left(O\left(\lambda^{-\frac{1}{2}}\right), O\left(\lambda^{\frac{1}{2}}\right)\right) d \lambda, \quad \lambda \rightarrow \infty
\end{aligned}
$$

on $\lambda>0$. The $O(\cdot)$-terms satisfy the natural derivative bounds.

Proof. Taking $\alpha=0$ and $x_{0}=0$ in (3.8) (and suppressing $x_{0}$ ) yields

$$
\theta_{0}(x ; z)=\theta_{0}(-x ; z), \quad \phi_{0}(x ; z)=-\phi_{0}(-x ; z), \quad \psi_{-, 0}(x ; z)=\psi_{+, 0}(-x ; z)
$$

whence $m_{-, 0}(z)=-m_{+, 0}(z)$ and $W(z)=2 m_{-, 0}(z)$. Denote by $u_{0,+}(x)$ and $u_{1,+}(x)$ a fundamental system of solutions to $\mathcal{L} f=0$ with

$$
\begin{aligned}
& u_{0,+}(x)=1+O\left(x^{-100}\right) \\
& u_{1,+}(x)=x+O\left(x^{-100}\right)
\end{aligned}
$$

as $x \rightarrow \infty$. This representation follows from the Volterra integral equations

$$
\begin{aligned}
& u_{0,+}(x)=1+\int_{x}^{\infty}(y-x) V(y) u_{0,+}(y) d y \\
& u_{1,+}(x)=x+\int_{x}^{\infty}(y-x) V(y) u_{1,+}(y) d y
\end{aligned}
$$

by iteration. In particular, $W\left(u_{1,+}, u_{0,+}\right)=1$. Furthermore, $u_{0,-}, u_{1,-}$ denote the corresponding solutions, but with $x \rightarrow-\infty$. By symmetry, $u_{0,-}(x)=u_{0,+}(-x)$ and $u_{1,-}(x)=-u_{1,+}(-x)$. Since zero energy is nonresonant, $W\left(u_{0,+}, u_{0,-}\right) \neq 0$. Perturbatively in $\lambda$, we now obtain from $u_{j,+}(x)$ unique eigenfunctions $u_{j,+}(x, \lambda)$ satisfying $\mathcal{L} u_{j,+}=\lambda u_{j,+}$, as well as for small $\lambda$ and $\left|\lambda x^{2}\right| \ll 1$,

$$
u_{j,+}(x, \lambda)=u_{j,+}(x)\left(1+O\left(\lambda x^{2}\right)\right) \quad j=0,1
$$

Indeed, $u_{j,+}(x, \lambda)$ are given in terms of the Volterra equations

$$
u_{j,+}(x, \lambda)=u_{j,+}(x)+\lambda \int_{0}^{x}\left[u_{0,+}(x) u_{1,+}(y)-u_{0,+}(y) u_{1,+}(x)\right] u_{j,+}(y, \lambda) d y
$$

Similarly, the Jost solutions $f_{+}(x, \lambda)$ defined by $\mathcal{L} f_{ \pm}(\cdot, \lambda)=\lambda f_{ \pm}(\cdot, \lambda), f_{ \pm}(x, \lambda) \simeq$ $e^{ \pm i x \lambda^{\frac{1}{2}}}$ as $x \rightarrow \pm \infty$, satisfy

$$
f_{ \pm}(x, \lambda)=e^{ \pm i x \lambda^{\frac{1}{2}}}\left(1+O\left(x^{-100}\right)\right) \quad \pm x \gg 1
$$


As usual, this follows from the Volterra representation of these functions. Moreover, one has

$$
f_{ \pm}(x, \lambda)=a_{ \pm}(\lambda) u_{0, \pm}(x, \lambda)+b_{ \pm}(\lambda) u_{1, \pm}(x, \lambda)
$$

with

$$
a_{ \pm}(\lambda)=-W\left(f_{ \pm}(\cdot, \lambda), u_{1, \pm}(\cdot, \lambda)\right), \quad b_{ \pm}(\lambda)=W\left(f_{ \pm}(\cdot, \lambda), u_{0, \pm}(\cdot, \lambda)\right)
$$

Therefore, for any small $\varepsilon>0$

$$
\begin{aligned}
& a_{ \pm}(\lambda)=1+O\left(\lambda^{1-\varepsilon}\right) \\
& b_{ \pm}(\lambda)=\mp i \lambda^{\frac{1}{2}}+O\left(\lambda^{1-\varepsilon}\right)
\end{aligned}
$$

as $\lambda \rightarrow 0$. In conclusion,

$$
W(\lambda):=W\left(f_{+}(\cdot, \lambda), f_{-}(\cdot, \lambda)\right)=c_{0}+i c_{1} \lambda^{\frac{1}{2}}+O\left(\lambda^{1-\varepsilon}\right)
$$

where $c_{0}, c_{1} \in \mathbb{R}$ with $c_{0} \neq 0$. The matrix in (3.10) is

$$
M_{0}(\lambda)=\operatorname{diag}\left(W(\lambda)^{-1}, \frac{1}{4} W(\lambda)\right)
$$

and the measure $\Omega_{0}(\lambda)$ satisfies, for small $\lambda$, by (3.12)

$$
\Omega_{0}(d \lambda)=\operatorname{diag}\left(O\left(\lambda^{\frac{1}{2}}\right), O\left(\lambda^{\frac{1}{2}}\right)\right) d \lambda, \quad \lambda \rightarrow 0
$$

For large $\lambda$, the free representation (3.15) describes $\Omega_{0}$ to leading order.

3.4. Evolution estimates. We now establish some estimates on the Klein-Gordon evolution relative to $L_{ \pm}$on the line. These are analogous to those for the Schrödinger evolution obtained in [14].

Lemma 3.5. Let $L_{ \pm}$be as in (3.1), (3.2), respectively, with $p>3$. Then one has the following bounds

$$
\begin{gathered}
\left\|\langle x\rangle^{-1} e^{ \pm i t L_{ \pm}^{\frac{1}{2}}} P_{c}\left(L_{ \pm}\right) f\right\|_{L_{x}^{\infty} L_{t}^{2}} \lesssim\left\|\left\langle\partial_{x}\right\rangle^{\frac{1}{2}} f\right\|_{2} \\
\left\|\langle x\rangle^{-1} \int_{-\infty}^{t} e^{ \pm i(t-s) L_{ \pm}^{\frac{1}{2}}} P_{c}\left(L_{ \pm}\right) f(s) d s\right\|_{L_{x}^{\infty} L_{t}^{2}} \lesssim \int_{-\infty}^{\infty}\left\|\left\langle\partial_{x}\right\rangle^{\frac{1}{2}} f(s)\right\|_{2} d s
\end{gathered}
$$

for all Schwartz functions $f$ on the line.

Proof. We begin with the first. Write $w(x)=\langle x\rangle^{-1}$. Then by duality we need to estimate, with $g=g(t, x)$, and $\mathcal{F}$ denoting the distorted Fourier transform of Proposition 3.2,

$$
\begin{aligned}
& \left\langle e^{ \pm i t L_{ \pm}^{\frac{1}{2}}} P_{c}\left(L_{+}\right) f \mid w g\right\rangle=\int_{-\infty}^{\infty} d t \int_{0}^{\infty} e^{ \pm i t(1+\lambda)^{\frac{1}{2}}} \mathcal{F} f(\lambda)^{T} \Omega_{0}(d \lambda) \mathcal{F}(w g(t))(\lambda) \\
& =\int_{-\infty}^{\infty} d t \int_{0}^{\infty} e^{ \pm i t(1+\lambda)^{\frac{1}{2}}} \mathcal{F}_{1} f(\lambda) \mu_{1}(\lambda) \int_{-\infty}^{\infty} w(x) g(t, x) \theta(x, \lambda) d x d \lambda \\
& +\int_{-\infty}^{\infty} d t \int_{0}^{\infty} e^{ \pm i t(1+\lambda)^{\frac{1}{2}}} \mathcal{F}_{2} f(\lambda) \mu_{2}(\lambda) \int_{-\infty}^{\infty} w(x) g(t, x) \phi(x, \lambda) d x d \lambda
\end{aligned}
$$


where $\Omega_{0}(d \lambda)=\operatorname{diag}\left(\mu_{1}, \mu_{2}\right) d \lambda$, and $\theta, \phi$ are $\theta_{0}(x, 0 ; z), \phi_{0}(x, 0 ; z)$ from above. Here $\lambda$ denotes the spectral variable of $\mathcal{L}=L_{ \pm}-1$. For small $\lambda$ one has

$$
(1+\lambda)^{\frac{1}{2}}=1+\frac{1}{2} \lambda+O\left(\lambda^{2}\right)
$$

Up to change of variable in $\lambda$ (which we ignore) we can therefore view the small $\lambda$ integral as the usual Fourier transform. Denoting the Fourier transform of $g(t, x)$ in time by $\widehat{g}(\lambda, x)$ we can bound the contribution of small $\lambda$ to (3.25) by means of (3.23) as follows:

$$
\begin{gathered}
\int_{0}^{1} \int_{-\infty}^{\infty}\left|\mathcal{F}_{j} f(\lambda)\right||\widehat{g}(\lambda, x)|[|\theta(x, \lambda)|+|\phi(x, \lambda)|] w(x) d x \mu_{j}(\lambda) d \lambda \\
\lesssim \sup _{0<\lambda<1} \sup _{x} w(x) \mu_{j}(\lambda)^{\frac{1}{2}}[|\theta(x, \lambda)|+|\phi(x, \lambda)|] \int_{-\infty}^{\infty}\left(\int_{0}^{1}\left|\mathcal{F}_{j} f(\lambda)\right|^{2} \mu_{j}(\lambda) d \lambda\right)^{\frac{1}{2}} \\
\quad \times\left(\int_{0}^{1}|\widehat{g}(\lambda, y)|^{2} d \lambda\right)^{\frac{1}{2}} d y \lesssim\|f\|_{2}\|g\|_{L_{x}^{1} L_{t}^{2}}
\end{gathered}
$$

In the final step we used the unitarity of the Fourier transform, both in the free and distorted cases, as well as the fact that uniformly in $\lambda>0$

$$
\sup _{x}\langle x\rangle^{-1}[|\theta(x, \lambda)|+|\phi(x, \lambda)|] \lesssim 1
$$

For $\lambda>1$, one has $(\lambda)^{\frac{1}{2}} \simeq \lambda^{\frac{1}{2}}$. Hence, the first component for these $\lambda$-values is bounded by (we can ignore $w(x)$ in this regime, as well as $\theta$, cf. (3.15)),

$$
\begin{aligned}
& \int_{1}^{\infty} \int_{-\infty}^{\infty}\left|\mathcal{F}_{1} f(\lambda)\right||\widehat{g}(\sqrt{\lambda}, x)| \mu_{1}(\lambda) d x d \lambda \\
& \lesssim \int_{1}^{\infty} \int_{-\infty}^{\infty}\left|\mathcal{F}_{1} f\left(\lambda^{2}\right)\right||\widehat{g}(\lambda, x)| \lambda \mu_{1}\left(\lambda^{2}\right) d x d \lambda \\
& \lesssim \int_{-\infty}^{\infty}\left(\int_{1}^{\infty}\left|\mathcal{F}_{1} f(\lambda)\right|^{2} \lambda \mu_{1}^{2}(\lambda) d \lambda\right)^{\frac{1}{2}}\left(\int_{1}^{\infty}|\widehat{g}(\lambda, x)|^{2} d \lambda\right)^{\frac{1}{2}} d x \\
& \lesssim\left\|\left\langle\partial_{x}\right\rangle^{\frac{1}{2}} f\right\|_{2}\|g\|_{L_{x}^{1} L_{t}^{2}}
\end{aligned}
$$

The final estimate here follows by the free asymptotics (3.15).

For the second inequality in the lemma one proceeds in a similar fashion. In fact, using the notation from the first part of the proof,

$$
\begin{aligned}
& \left\langle\int_{-\infty}^{t} e^{ \pm i(t-s) L_{ \pm}^{\frac{1}{2}}} P_{c}\left(L_{ \pm}\right) f(s) d s \mid w g\right\rangle \\
& =\int_{-\infty}^{\infty} d t \int_{-\infty}^{t} d s \int_{-\infty}^{\infty} e^{ \pm i(t-s)(1+\lambda)^{\frac{1}{2}}} \mathcal{F}(f(s))(\lambda)^{T} \Omega_{0}(d \lambda) \mathcal{F}(w g(t))(\lambda) \\
& =\int_{-\infty}^{\infty} d s \int_{s}^{\infty} d t \int_{0}^{\infty} e^{ \pm i(t-s)(1+\lambda)^{\frac{1}{2}}} \mathcal{F}_{1}(f(s))(\lambda) \mu_{1}(\lambda) \int_{-\infty}^{\infty} w(x) g(t, x) \theta(x, \lambda) d x d \lambda \\
& +\int_{-\infty}^{\infty} d s \int_{s}^{\infty} d t \int_{0}^{\infty} e^{ \pm i(t-s)(1+\lambda)^{\frac{1}{2}}} \mathcal{F}_{2}(f(s))(\lambda) \mu_{2}(\lambda) \int_{-\infty}^{\infty} w(x) g(t, x) \phi(x, \lambda) d x d \lambda
\end{aligned}
$$


Carrying out the $t$-integration, and performing similar arguments as in the previous case, shows that the two final expressions here are

$$
\lesssim \int\left\|\left\langle\partial_{x}\right\rangle^{\frac{1}{2}} f(s)\right\|_{2} d s\|g\|_{L_{x}^{1} L_{t}^{2}}
$$

as desired.

Note that (3.24) cannot hold for the free case, i.e., $e^{i t\left\langle\partial_{x}^{2}\right\rangle^{\frac{1}{2}}}$ since the best point-wise decay of the latter evolution is $t^{-\frac{1}{2}}$. For the nonlinear analysis, it will be technically advantageous to work in $L_{x}^{2}$ rather than with $L_{x}^{\infty}$ on the left-hand side of (3.24). This can easily be done just by switching to faster decaying weights. In addition, we shall add half of a derivative to the left-hand side, reflecting the fact that we are working in the energy space $H^{1}$. As standard derivatives do not commute with $L_{+}$, this step requires some care. While one could use powers of $L_{+}$, via Lemma 3.3, we instead rely on some algebra involving $L_{-}$. The precise statement is as follows. Recall that $\rho$ is the ground state of $L_{+}$, and that $Q^{\prime}$ satisfies $L_{+} Q^{\prime}=0$.

Corollary 3.6. Any solution of

$$
\ddot{u}=-L_{+} u+F, \quad u \perp \rho, Q^{\prime}
$$

satisfies

$$
\left\|\langle x\rangle^{-s} u\right\|_{L_{t}^{2} H_{x}^{1 / 2}} \lesssim\|u(0)\|_{H^{1}}+\|\dot{u}(0)\|_{L^{2}}+\|F\|_{L_{t}^{1} L_{x}^{2}}
$$

with $s>\frac{3}{2}$.

Proof. By Lemma 3.5, any $u$ as in (3.28) satisfies

$$
\left\|\langle x\rangle^{-1} u\right\|_{L_{x}^{\infty} L_{t}^{2}} \lesssim\|u(0)\|_{H^{1 / 2}}+\|\dot{u}(0)\|_{H^{-1 / 2}}+\|F\|_{L_{t}^{1} H^{-1 / 2}}
$$

The left-hand side dominates

$$
\left\|\langle x\rangle^{-s} u\right\|_{L_{t}^{2} L_{x}^{2}} \quad(s>3 / 2) .
$$

Similarly, any solution of $\ddot{v}=-L_{-} v+\tilde{F}$ with $v \perp Q$ satisfies

$$
\left\|\langle x\rangle^{-s} v\right\|_{L_{t, x}^{2}} \lesssim\|v(0)\|_{H^{1 / 2}}+\|\dot{v}(0)\|_{H^{-1 / 2}}+\|\tilde{F}\|_{L_{t}^{1} H^{-1 / 2}} .
$$

In order to estimate the derivative, we use the special one-dimensional property

$$
U:=\rho \partial_{x} \rho^{-1}, \quad \rho=C Q^{(p+1) / 2}, \quad U L_{+}=L_{-} U, \quad U^{*} Q=-C Q_{x},
$$

where $C$ denotes positive constants dependent on $p$.

In particular, with $u$ as before, $v:=U u$ and $\tilde{F}=U F$ satisfies (3.32), whence

$$
\begin{aligned}
\left\|\langle x\rangle^{-s} u\right\|_{L_{t}^{2} H_{x}^{1}} \lesssim\left\|\langle x\rangle^{-s} u\right\|_{L_{t, x}^{2}}+\left\|\langle x\rangle^{-s} U u\right\|_{L_{t, x}^{2}} \\
\lesssim\|u(0)\|_{H^{1 / 2}}+\|\dot{u}(0)\|_{H^{-1 / 2}}+\|U u(0)\|_{H^{1 / 2}}+\|U \dot{u}(0)\|_{H^{-1 / 2}} \\
\quad \quad+\|F\|_{L_{t}^{1} H^{-1 / 2}}+\|\tilde{F}\|_{L_{t}^{1} H^{-1 / 2}} \\
\vdots\|u(0)\|_{H^{3 / 2}}+\|\dot{u}(0)\|_{H^{1 / 2}}+\|F\|_{L_{t}^{1} H^{1 / 2}}
\end{aligned}
$$

Interpolating it with the estimate without the derivative yields

$$
\left\|\langle x\rangle^{-s} u\right\|_{L_{t}^{2} H_{x}^{1 / 2}} \lesssim\|u(0)\|_{H^{1}}+\|\dot{u}(0)\|_{L^{2}}+\|F\|_{L_{t}^{1} L_{x}^{2}}
$$

as claimed. 
The significance of (3.29) lies with the perturbative nonlinear analysis of Section 3.5. In fact, placing a nonlinear term such as $Q^{q} u^{2}$ in $L_{t}^{1} L_{x}^{2}$ yields

$$
\left\|Q^{q} u^{2}\right\|_{L_{t}^{1} L_{x}^{2}} \lesssim\left\|\langle x\rangle^{-s} u\right\|_{L_{t}^{2} L_{x}^{4}}^{2} \lesssim\left\|\langle x\rangle^{-s} u\right\|_{L_{t}^{2} H_{x}^{\frac{1}{2}}}^{2}
$$

for $H^{1}$ solutions $u$. This will allows us to close our estimates very easily.

We shall also require Strichartz estimates on the Klein-Gordon equation relative to $\mathcal{L}$. Note that unlike Lemma 3.5, Corollary 3.7 has nothing to do with $\mathcal{L}$ having a zero energy resonance or not.

Corollary 3.7. For any Schwartz function $u$ in $\mathbb{R}_{t, x}^{1+1}$ with $u=P_{c}(\mathcal{L}) u$ one has the aprori bound

$$
\|u\|_{L_{t}^{p} L_{x}^{r}} \lesssim\|u[0]\|_{H^{1} \times L^{2}}+\left\|\ddot{u}+L_{ \pm} u\right\|_{L_{t}^{1} L_{x}^{2}}
$$

for any $4<p \leq \infty, 0<\frac{1}{r} \leq \frac{1}{2}-\frac{2}{p}$. In particular, one can take $r=2 p$ for any $5 \leq p<\infty$.

Proof. We first recall the following Strichartz estimates for the free case, see for example [8, Section 4.2]: for any Schwartz function $u$ in $\mathbb{R}_{t, x}^{1+1}$ there is the aprori bound

$$
\|u\|_{L_{t}^{4} B_{\infty, 2}^{\frac{1}{4}}}+\|u\|_{L_{t}^{\infty} H^{1}}+\|\dot{u}\|_{L^{\infty} L^{2}} \lesssim\|u[0]\|_{H^{1} \times L^{2}}+\|\square u+u\|_{L_{t}^{\frac{4}{3}} B_{1,2}^{\frac{3}{4}}+L_{t}^{1} L_{x}^{2}}
$$

Here $B_{p, 2}^{\sigma}$ are the usual inhomogeneous Besov spaces. By interpolation, one obtains the following bounds: for any $4 \leq p \leq \infty$, and any $2 \leq q \leq \infty$,

$$
\|u\|_{L_{t}^{p} B_{q, 2}^{\alpha}} \lesssim\|u[0]\|_{H^{1} \times L^{2}}+\|\square u+u\|_{L_{t}^{\frac{4}{3}} B_{1,2}^{\frac{3}{4}}+L_{t}^{1} L_{x}^{2}}
$$

where $\alpha=1-\frac{3}{p}, \frac{1}{q}=\frac{1}{2}-\frac{2}{p}$. By the embedding $B_{q, 2}^{\alpha} \hookrightarrow L^{r}(\mathbb{R})$ for any $q \leq r<\infty$ provided $\alpha \geq \frac{1}{q}-\frac{1}{r}$, we now conclude that

$$
\|u\|_{L_{t}^{p} L_{x}^{r}} \lesssim\|u[0]\|_{H^{1} \times L^{2}}+\|\square u+u\|_{L_{t}^{\frac{4}{3}} B_{1,2}^{\frac{3}{4}}+L_{t}^{1} L_{x}^{2}}
$$

for any $4<p \leq \infty, 0<\frac{1}{r} \leq \frac{1}{2}-\frac{2}{p}$. Finally, (3.37) follows from (3.40) and the $L^{r}$-boundedness of the wave operators of $\mathcal{L}$ for $1<r<\infty$.

The condition $p \geq 5$ is significant for the Strichartz-based small data scattering theory: indeed, consider the equation

$$
\square u+u= \pm|u|^{p-1} u
$$

on the line. Then placing the nonlinearity in $L_{t}^{1} L_{x}^{2}$ requires Strichartz control on $\|u\|_{L_{t}^{p} L_{x}^{2 p}}$. However, the latter means that we need $\frac{1}{2 p} \leq \frac{1}{2}-\frac{2}{p}$, or $p \geq 5$ (the latter being the $L^{2}$-critical power in dimension 1).

3.5. Proof of Proposition 3.1. We shall now show that for even functions one can construct the center-stable manifold for

$$
\square u+u=|u|^{p-1} u
$$

in 1-dim where $p>5$ is fixed. Writing $u=Q+v$ with $v$ small, one has

$$
\ddot{v}+L_{+} v=N(Q, v)
$$


where $L_{+}=-\partial_{x}^{2}+1-p Q^{p-1}$. The nonlinearity $N$ satisfies the bound

$$
|N(Q, v)| \lesssim Q^{p-2}|v|^{2}+|v|^{p}
$$

The operator $L_{+}$has negative spectrum, $L_{+} \rho=-k^{2} \rho$ and one therefore has to write $v(t, x)=\mu(t) \rho+w(t, x)$. The resulting equations are

$$
\begin{aligned}
\left(\ddot{\mu}-k^{2} \mu\right)(t) \rho & =P_{\rho} N(Q, v) \\
\ddot{w}+P_{\rho}^{\perp} L_{+} w & =P_{\rho}^{\perp} N(Q, v)
\end{aligned}
$$

To control the $w$-equation we shall use the Strichartz norm

$$
\|w\|_{S}:=\|(w, \dot{w})\|_{H^{1} \times L^{2}}+\|w\|_{L_{t}^{p} L_{x}^{2 p}}
$$

as well as the localized norm

$$
\|w\|_{L}:=\left\|\langle x\rangle^{-2} w\right\|_{L_{t, x}^{2}}
$$

We now close the estimates for $w$ in the space $\|w\|_{X}=\|w\|_{S}+\|w\|_{L}$, and those for $\mu$ in the space $L^{1} \cap L^{\infty}([0, \infty))$. Applying the Strichartz estimates for $L_{+}$and using (3.36) yields

$$
\|w\|_{S} \lesssim\|w[0]\|_{\mathcal{H}}+\|w\|_{L}^{2}+\|w\|_{S}^{p} \lesssim \delta+\|w\|_{X}^{2}
$$

where $\|w[0]\|_{\mathcal{H}}<\delta<\nu$. Note that we are assuming the desired bound on $v$ appearing on the right-hand side of the equation, in keeping with the usual contraction argument setup. To bound the local norm, we use Corollary 3.6 to conclude that

$$
\left\|\langle x\rangle^{-2} w\right\|_{L_{t, x}^{2}} \lesssim\|\tilde{v}[0]\|_{\mathcal{H}}+\|N(Q, v)\|_{L_{t}^{1} L_{x}^{2}}
$$

which can be estimated as before. Combining (3.47) with (3.48) yields

$$
\|w\|_{X} \lesssim \delta+\|v\|_{X}^{2}
$$

For the $\mu$ equation in (3.44) we introduce

$$
\mu_{ \pm}=\frac{1}{2}\left(\mu \pm \frac{1}{k} \dot{\mu}\right), \quad \mu=\frac{1}{2}\left(\mu_{+}+\mu_{-}\right)
$$

which implies that

$$
\begin{aligned}
\dot{\mu}_{ \pm} & =\frac{1}{2}(\dot{\mu} \pm k \mu) \pm \frac{1}{2 k} P_{\rho} N(Q, v) \\
& = \pm k \mu_{ \pm} \pm \frac{1}{2 k} P_{\rho} N(Q, v)
\end{aligned}
$$

The homogeneous solutions are $\mu_{ \pm}(t)=e^{ \pm k t}$. Under the stability condition

$$
0=\mu_{+}(0)+\frac{1}{2 k} \int_{0}^{\infty} e^{-k s} P_{\rho} N(Q, v)(s) d s
$$

the solutions to (3.50) are

$$
\begin{aligned}
& \mu_{+}(t)=-\frac{1}{2 k} \int_{t}^{\infty} e^{-k(s-t)} P_{\rho} N(Q, v)(s) d s \\
& \mu_{-}(t)=e^{-k t} \mu_{-}(0)+\frac{1}{2 k} \int_{0}^{t} e^{-k(t-s)} P_{\rho} N(Q, v)(s) d s
\end{aligned}
$$


Therefore, assuming (3.51), one obtains the estimates

$$
\begin{aligned}
\|\mu\|_{L^{1} \cap L^{\infty}((0, \infty))} & \lesssim\left|\mu_{-}(0)\right|+\int_{0}^{\infty}|\langle N(Q, v)(s), \rho\rangle| d s \\
& \lesssim \delta+\|N(Q, v)\|_{L_{t}^{1} L_{x}^{2}} \lesssim \delta+\|v\|_{X}^{2}
\end{aligned}
$$

It is now straightforward to show that the iteration (or, the fixed-point argument) yields a unique solution $(\mu, w) \in X_{0} \times X$, where $X_{0}=L^{1} \cap L^{\infty}((0, \infty))$ and $X$ is as before, with $\|(\mu, w)\|_{X_{0} \times X} \lesssim \delta$. Moreover, $w$ scatters with scattering data

$$
w_{\infty}:=w(0)+\int_{0}^{\infty} \frac{\sin (t \omega)}{\omega} N_{\rho}^{\perp}(Q, v)(t) d t
$$

where $\omega:=\left(P_{c}\left(L_{+}\right) L_{+}\right)^{\frac{1}{2}}$.

For the uniqueness, we note that if $(\mu, w)$ are such that

$$
\sup _{t \geq 0}\left[|\mu(t)|+|\dot{\mu}(t)|+\|w(t)\|_{H^{1}}+\|\dot{w}(t)\|_{L^{2}}\right] \lesssim \delta
$$

then necessarily (3.51) holds, whence also (3.52). Denoting for any finite $T>0$

$$
\|(\mu, w)\|_{X_{T}}:=\|\mu\|_{L^{1}(0, T)}+\|\mu\|_{L^{\infty}(T, \infty)}+\|w\|_{S(0, T)}
$$

we thus conclude from the integral equations that

$$
\sup _{T>0}\|(\mu, w)\|_{X_{T}} \lesssim \delta
$$

Hence also $\|(\mu, w)\|_{X} \lesssim \delta$ and one obtains the claim from the uniqueness of the fixed point. Finally, (3.4) holds since the distance on the left-hand side of that inequality is proportional to $\mu_{+}(0)$ as given by (3.51). Since the integral in the expression is of size $O\left(\delta^{2}\right)$, we are done.

3.6. Proof of Theorem $\mathbf{1 . 1}$ and Corollary 1.2. We only need to address the behavior of those solutions in Theorem 2.1 which are trapped as $t \rightarrow \infty$. If so, then the uniqueness part of Proposition 3.1 states that $\vec{u}(t)$ lies on the manifold $\mathcal{M}$ constructed in that proposition for all large $t$. Therefore, the set of all data which lead to solutions trapped by $Q$ in forward times is the maximal backward evolution of $\mathcal{M}$ under the flow of (1.1). This can easily be seen to be a $C^{1}$ manifold (in fact, it has better smoothness). The scattering to $Q$ was shown in Proposition 3.1, which concludes the proof of Theorem 1.1.

For the corollary, we proceed as in [15]. Thus, if $E(\vec{u})=E(Q, 0)$ and if the solution is trapped, then $\vec{w}_{\infty}=0$. Therefore, the entire dynamics is controlled by a single quantity, namely $\mu_{-}(t)$. This in turn is determined uniquely by $\mu_{-}(0)$. It is clear that $\mu_{-}(0)=0$ forces $\mu_{-} \equiv 0$, or in other words, $u=Q$ for all times. If $\mu_{-}(0) \neq$ 0 , then it can never change sign which leads to the two solutions $W_{ \pm}$. Since these are clearly one-dimensional and time-translation is a symmetry for these special solutions, they are all obtained by time-translation of two fixed representatives of solutions converging to $Q$. Since the sign of $K_{0}$ is the same as that of $-\mu_{-}$ upon ejection from a neighborhood of $(Q, 0)$, we see that $W_{ \pm}$have the behavior as $t \rightarrow-\infty$ as described in Corollary 1.2. 


\section{REFERENCES}

[1] Artbazar, G., Yajima, K. The $L^{p}$-continuity of wave operators for one dimensional Schrödinger operators. J. Math. Sci. Univ. Tokyo 7 (2000), no. 2, 221-240.

[2] Bates, P. W., Jones, C. K. R. T. Invariant manifolds for semilinear partial differential equations. Dynamics reported, Vol. 2, 1-38, Dynam. Report. Ser. Dynam. Systems Appl., 2, Wiley, Chichester, 1989.

[3] Bahouri, H., Gérard, P. High frequency approximation of solutions to critical nonlinear wave equations. Amer. J. Math. 121 (1999), no. 1, 131-175.

[4] Cuccagna, S. On asymptotic stability in energy space of ground states of NLS in $1 D$. J. Differential Equations 245 (2008), no. 3, 653-691.

[5] Duyckaerts, T., Merle, F. Dynamic of threshold solutions for energy-critical NLS. Geom. Funct. Anal. 18 (2009), no. 6, 1787-1840.

[6] Duyckaerts, T., Merle, F. Dynamics of threshold solutions for energy-critical wave equation. Int. Math. Res. Pap. IMRP 2008

[7] Gesztesy, F., Zinchenko, M. On spectral theory for Schrödinger operators with strongly singular potentials. Math. Nachr. 279 (2006), no. 9-10, 1041-1082.

[8] Ibrahim, S., Masmoudi, N., Nakanishi, K. Scattering threshold for the focusing nonlinear Klein-Gordon equation, to appear in Journal Analysis and PDEs

[9] Kenig, C., Merle, F. Global well-posedness, scattering and blow-up for the energy-critical, focusing, non-linear Schrödinger equation in the radial case. Invent. Math. 166 (2006), no. 3, $645-675$

[10] Kenig, C., Merle, F. Global well-posedness, scattering and blow-up for the energy-critical focusing non-linear wave equation. Acta Math. 201 (2008), no. 2, 147-212.

[11] Krieger, J., Schlag, W. Stable manifolds for all monic supercritical focusing nonlinear Schrödinger equations in one dimension. J. Amer. Math. Soc. 19 (2006), no. 4, 815-920.

[12] Krieger, J., Nakanishi, K., Schlag, W. Global dynamics away from the ground state for the energy-critical nonlinear wave equation, preprint 2010.

[13] Merle, F., Vega, L. Compactness at blow-up time for $L^{2}$ solutions of the critical nonlinear Schrödinger equation in 2D. Internat. Math. Res. Notices 1998, no. 8, 399-425.

[14] Mizumachi, T. Asymptotic stability of small solitary waves to $1 D$ nonlinear Schrödinger equations with potential. J. Math. Kyoto Univ. 48 (2008), no. 3, 471-497.

[15] Nakanishi, K., Schlag, W. Global dynamics above the ground state energy for the focusing nonlinear Klein-Gordon equation, preprint 2010.

[16] Nakanishi, K., Schlag, W. Global dynamics above the ground state energy for the cubic NLS equation in 3D, preprint 2010.

[17] Nakanishi, K., Schlag, W. Global dynamics above the ground state for the nonlinear KleinGordon equation without a radial assumption, preprint 2010.

[18] Payne, L. E., Sattinger, D. H. Saddle points and instability of nonlinear hyperbolic equations. Israel J. Math. 22 (1975), no. 3-4, 273-303.

[19] Weder, R. The $W_{k, p}$-continuity of the Schrödinger wave operators on the line. Comm. Math. Phys. 208 (1999), no. 2, 507-520.

BÂtiment des Mathématiques, Station 8, CH-1015 Lausanne, Suisse

E-mail address: joachim.krieger@epfl.ch

Department of Mathematics, Kyoto University, Kyoto 606-8502, Japan

E-mail address: n-kenji@math.kyoto-u.ac.jp

Department of Mathematics, The University of Chicago, Chicago, IL 60615, U.S.A.

E-mail address: schlag@math.uchicago.edu 